\title{
BMJ Open Development of salient medication reminders to facilitate information transfer during transition from inpatient to primary care: the Delphi process
}

To cite: Wong EL-Y, Tang K$S$, Cheung AW-L, et al. Development of salient medication reminders to facilitate information transfer during transition from inpatient to primary care: the Delphi process. BMJ Open 2021;11:e041336. doi:10.1136/ bmjopen-2020-041336

- Prepublication history and additional material for this paper is available online. To view these files, please visit the journal online (http://dx.doi.org/10. 1136/bmjopen-2020-041336).

Received 05 June 2020 Revised 09 March 2021 Accepted 17 March 2021

Check for updates

(c) Author(s) (or their employer(s)) 2021. Re-use permitted under CC BY-NC. No commercial re-use. See rights and permissions. Published by BMJ.

For numbered affiliations see end of article.

Correspondence to Professor Eliza Lai-Yi Wong; lywong@cuhk.edu.hk

\section{ABSTRACT}

Objective Transitional care is important to successful hospital discharge. Providing patients with a clear and concise summary of medication-related information can help improve outcomes, in particular, among older adults. The present study aimed to propose a framework for the development of salient medication reminders (SMR), which include drug-related risks and precautions, using the Delphi process.

Design Identification of potential SMR statements for $80 \%$ of medication types used by older adult patients discharged from geriatric medicine departments, followed by a Delphi survey and expert panel discussion.

Settings Medical and geriatric departments of public hospitals in Hong Kong.

Participants A panel of 13 geriatric medical experts. Outcome measure A Likert scale ranging from 1 (strongly disagree) to 5 (strongly agree) points, scoring item relevance, importance and clarity. The minimum of $70 \%$ consensus was required for each statement to be included. Results The expert panel achieved consensus through the Delphi process on 80 statements for 44 medication entities. Subsequently, the SMR steering group endorsed the inclusion of these statements in the SMR to be disseminated among older adults at the time of discharge from geriatric medicine departments.

Conclusions The Delphi process contributed to the development of SMR for older adult patients discharged from public hospitals in Hong Kong. Patient experience with and staff response to the SMR were assessed at four hospitals before implementation at all public hospitals.

\section{INTRODUCTION}

Medication-related adverse events are a significant and often preventable cause of morbidity and mortality. ${ }^{1}$ Older adults are susceptible to medication-related harm due to polypharmacy, low health literacy and age-related limitations. ${ }^{2}$ Non-adherence is a component of medication-related harm
Strengths and limitations of this study

- Delphi-based consensus was used to identify 80 statements covering 24 drug classes most commonly dispensed to older adults in the public healthcare system in Hong Kong. The proposed framework may serve as a reference for the development of medication reminders aimed at improving patient experience and safety.

- The expert panel and steering committee included experts from geriatric medicine and other disciplines to ensure older adult care pathways were represented in the development of medication reminders.

- The Delphi survey provided opportunities for experts to deepen their understanding of pertinent issues and further refine the relevant statements.

- Expert discussion was held at the end of the Delphi process, helping prevent confrontations and ensure participant anonymity, both of which were conducive to a free discussion, increasing the ownership and acceptance of the salient medication reminder statements.

- Input from other stakeholder groups such as frontline workers and patients was not considered in the present study and should be incorporated in future studies.

among older adults that may experience difficulty in managing complex drug regimens for their multimorbidity. ${ }^{3}$ A systematic review has reported that the incidence of medication-related harm among patients aged $\geq 65$ years was in the range of $0.4 \%-$ $51.2 \%$, while $35 \%-59 \%$ of these incidents were likely preventable. ${ }^{2}$ A study from the UK using large-scale secondary data revealed that $37 \%$ of older adults experienced medicationrelated harm, and $81 \%$ of them experienced serious events; four patients died as a 
result. ${ }^{2}$ The incidence of hospitalisations associated with medication-related harm is 78 per 1000 discharges. $^{2}$

Five classes of medications are associated with the highest risk of medication-related harm, namely, opiates, antibiotics, benzodiazepines and antihypertensive and cardiovascular medicines, all of which are commonly used..$^{24}$ The WHO Global Patient Safety Challenge aims to reduce the incidence of preventable medication-related harm by $50 \%$ in the next 5 years. ${ }^{5}$ Information transfer at hospital discharge plays a vital role in achieving this goal; however, little is known about how this transfer can be completed effectively.

Hospital discharge is not equivalent with the end of care; rather, it is a transition step between acute care and primary care in an ambulatory setting either at home or an assisted living facility. ${ }^{5}$ This process may entail changes to medication; in such cases, incomplete information or ineffective communication at discharge may result in adverse events, ${ }^{67}$ most of which can be prevented or reversed, provided the patient or their caregiver have the right information. ${ }^{8}$ Patients and their carers need to be provided with information on the possible medicationrelated benefits and side effects, so that ambulatory or primary care can be provided effectively and with continuity, leading to desirable outcomes. Patients with limited knowledge on the risk of adverse events associated with their prescription may experience poor outcomes after discharge. Previous studies have shown that the period of care transition makes patients particularly susceptible to medication-related harm. ${ }^{4}$ On discharge, patients should be equipped with information on their regimen, including the recommended precautions. ${ }^{9}{ }^{10}$ However, effective communication in this context has rarely been studied, limiting the available evidence to reports of patient experience at discharge.

The Hong Kong Government has estimated the prevalence of chronic conditions requiring long-term medication at $70 \%$ among older adults; at least $40 \%$ of the affected adults have comorbidities. In addition, although approximately $25 \%$ of older adults require informal care, less than half $(47 \%)$ of them have caregivers. ${ }^{11}$ Polypharmacy is prevalent, accounting for approximately half of institutionalised older adults. Providing patients with clear instructions regarding their care may help improve these trends. ${ }^{11}$

Perceived adverse effects of medication are among the risk factors for non-adherence among the Chinese older adults, ${ }^{12}$ in particular, those without family or community support. ${ }^{13}$ This group is likely to benefit most from clear and concise summary of information on their prescriptions. Such summaries may also facilitate information transfer from healthcare providers to formal and informal caregivers, alongside patients themselves.

Transition of care quality is reflected indirectly in patient-reported experience. The 2019 Hong Kong Inpatient Experience Survey reported that $93 \%$ of patients

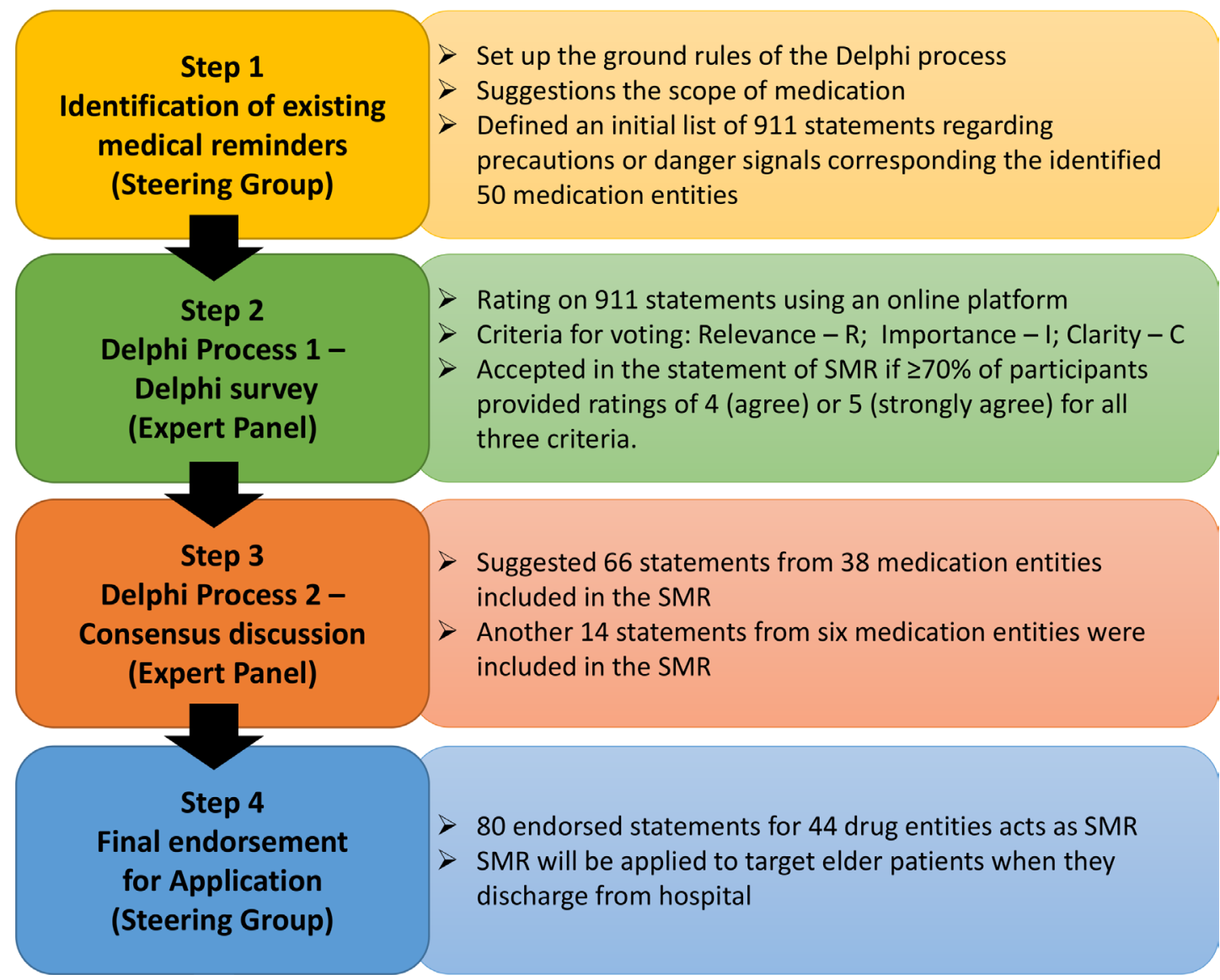

Figure 1 Study flow. SMR, salient medication reminder. 


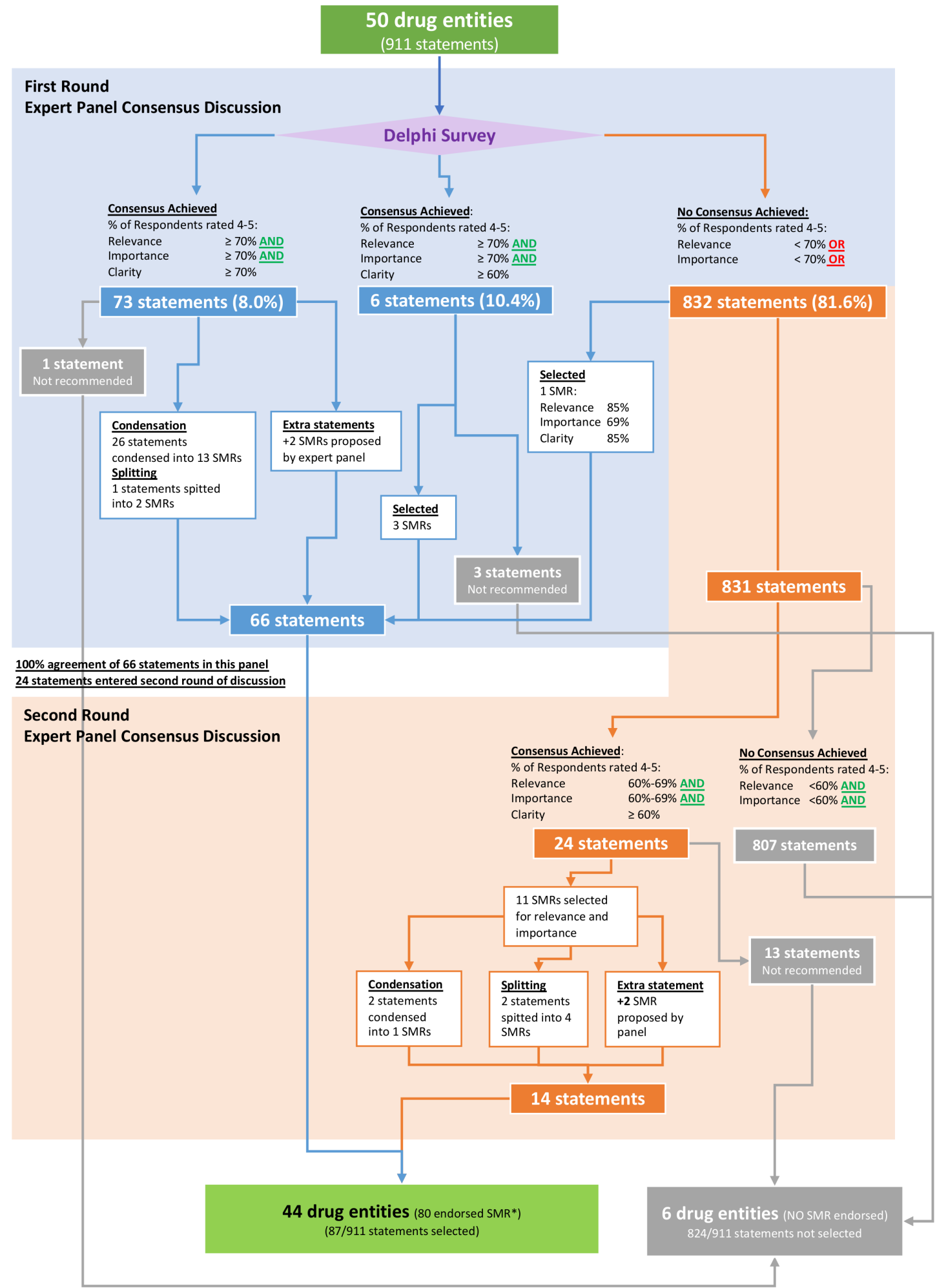

Figure 2 Results of Delphi process. SMRs, salient medication reminder.

had received on discharge clear and understandable information on how to take medication at home; however, approximately one-third of patients did not receive any information regarding treatment side effects or recommended precautions. ${ }^{4}$ This finding may be due to information overload or complexity. One local inpatient study suggested that $6 \%$ of hospital readmissions is due to side effects of drugs/drug-drug interaction. ${ }^{15}$ Other research suggests that discharge planning and postdischarge support may reduce readmission rates and improve health outcomes. ${ }^{16}$

Effectively communicating drug-related risks may reduce medication-related harm, concurrently allowing for more effective use of public resources by preventing 


\begin{tabular}{lllll} 
Table 1 & Demographics of expert panel members & & \\
Name & Gender & Post & Specialty & HA cluster \\
\hline Expert 1 & $\mathrm{M}$ & Consultant & Medicine and geriatrics & Cluster 7 \\
Expert 2 & $\mathrm{M}$ & Associate consultant & Medicine & Cluster 1 \\
Expert 3 & $\mathrm{M}$ & Deputy consultant & Medicine and geriatrics & Cluster5 \\
Expert 4 & $\mathrm{M}$ & Consultant & Medicine & Cluster 1 \\
Expert 5 & $\mathrm{M}$ & Consultant & Medicine & Cluster 3 \\
Expert 6 & $\mathrm{F}$ & Consultant & Medicine and geriatrics & Cluster 6 \\
Expert 7 & $\mathrm{M}$ & Consultant & Medicine and geriatrics & Cluster 5 \\
Expert 8 & $\mathrm{F}$ & Consultant & Medicine and geriatrics/ & Cluster 4 \\
Expert 9 & & Intensive-care Unit & Cluster 6 \\
Expert 10 & $\mathrm{M}$ & Associate consultant & Medicine & Cluster 2 \\
Expert 11 & $\mathrm{M}$ & Consultant & Geriatrics & Cluster 7 \\
Expert 12 & $\mathrm{M}$ & Deputy consultant & Medicine and geriatrics & Cluster 3 \\
Expert 13 & $\mathrm{M}$ & Associate consultant & Medicine and geriatrics & Cluster 4
\end{tabular}

F, female; HA, Hospital Authority; M, male.

medication-related readmissions. This evidence suggests the importance of improving information resources provided to patients on discharge, ensuring end-user awareness of treatment benefits and side effects and recommended precautions. The present study aimed to use the Delphi process to develop a framework for communicating precautions through the salient medication reminder (SMR) for older adults discharged from hospital. The present study is first to propose a medication reminder aimed at older adults discharged from geriatric medicine departments. The present framework may serve as a reference point for other health systems and for further research aimed at improving patient experience and safety.

\section{METHODS}

The study comprised four phases: (1) identifying and shortlisting candidate statements for the SMR by the steering group, (2) conducting a Delphi survey with an expert panel, (3) holding two rounds of consensus discussion among the experts and (4) seeking final endorsement from the steering group for the proposed SMR items.

The most common medication entities were identified and selected by the SMR steering group based on the medical records of previously discharged patients and previous reports of safety incidents. In addition, the steering group performed a Delphi survey of the statements shortlisted by the expert panel, which was followed by discussion. The Delphi process refers to structured consensus building among a diverse group of experts. ${ }^{17-19}$ The final selection and modification of the reminder statements would act as an SMR framework for future development in different types of inpatient discharge drugs. The study flow chart is presented in figure 1 .

\section{Study setting and participants}

The steering group consisted of 12 persons: two representatives from the patient experience survey research team at The Chinese University of Hong Kong, two representatives from the Division of Quality and Safety of the Hospital Authority (HA), and eight HA healthcare professional representatives, including three doctors, two nurses and three pharmacists.

The expert panel consisted of healthcare professionals with at least 10 years of experience in the field of geriatric medicine, including at least one expert from each of the seven geographical clusters that fall under the HA. A blind response was used for the Delphi survey and a consensus discussion. The experts submitted their responses via an online platform designed for the Delphi survey. Where response clarification was required, the research team followed up with the respondents over the phone. Subsequently, the experts met for a consensus discussion.

\section{Candidate medication statements}

The medication database provided information on side effects and warning signs associated with most drug entities commonly dispensed at hospitals. ${ }^{20}$ The steering group included medication entities reported in the 2017 medication profiles of patients discharged from geriatric medicine departments of large-scale acute care public hospitals in HA as well as those reported in previous safety incident records. A total of 50 medication entities were identified, covering approximately $80 \%$ of medication types used for older adult patients. These medication entities with a total of 911 statements on recommended precautions or danger signals were extracted for the Delphi survey. 


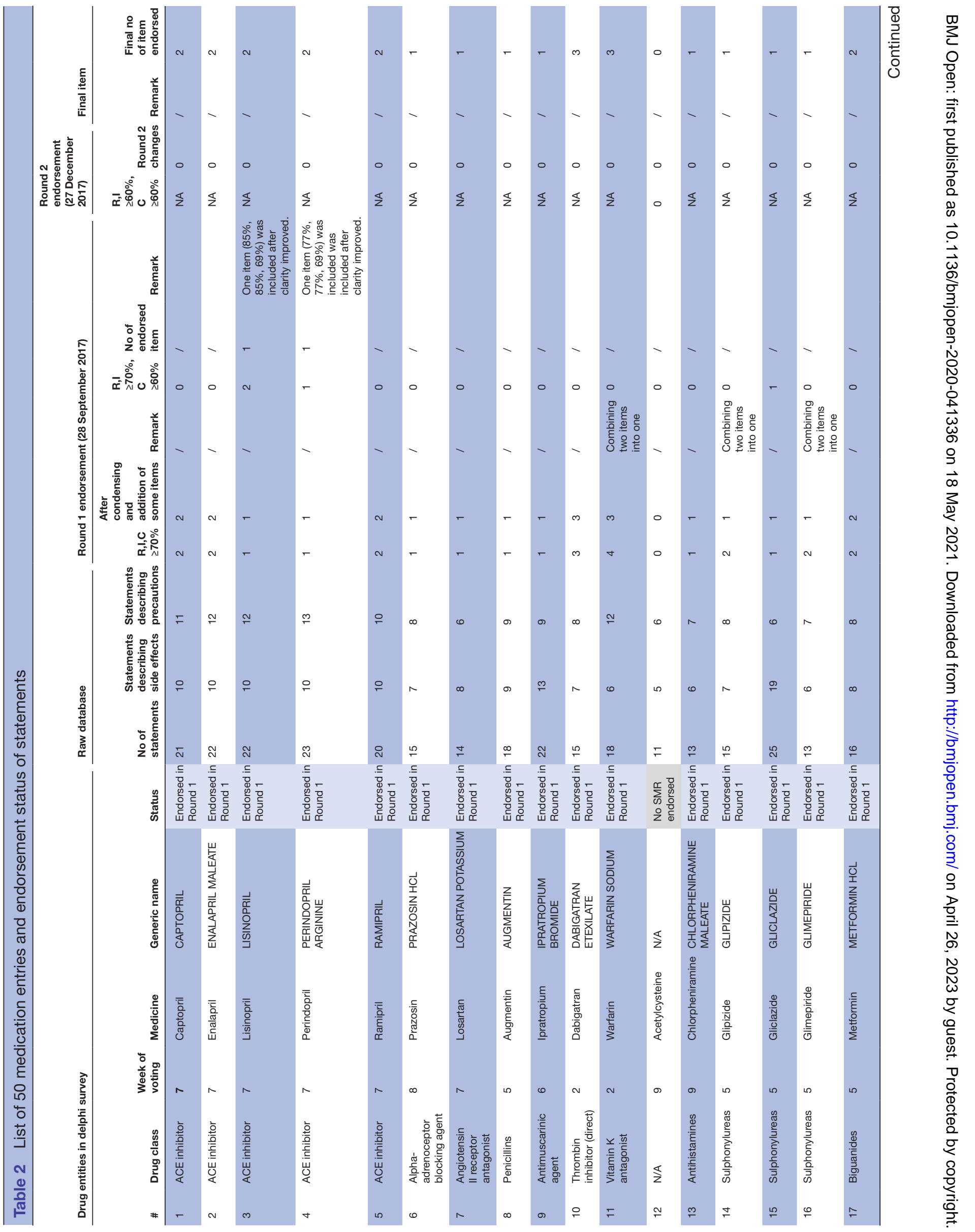




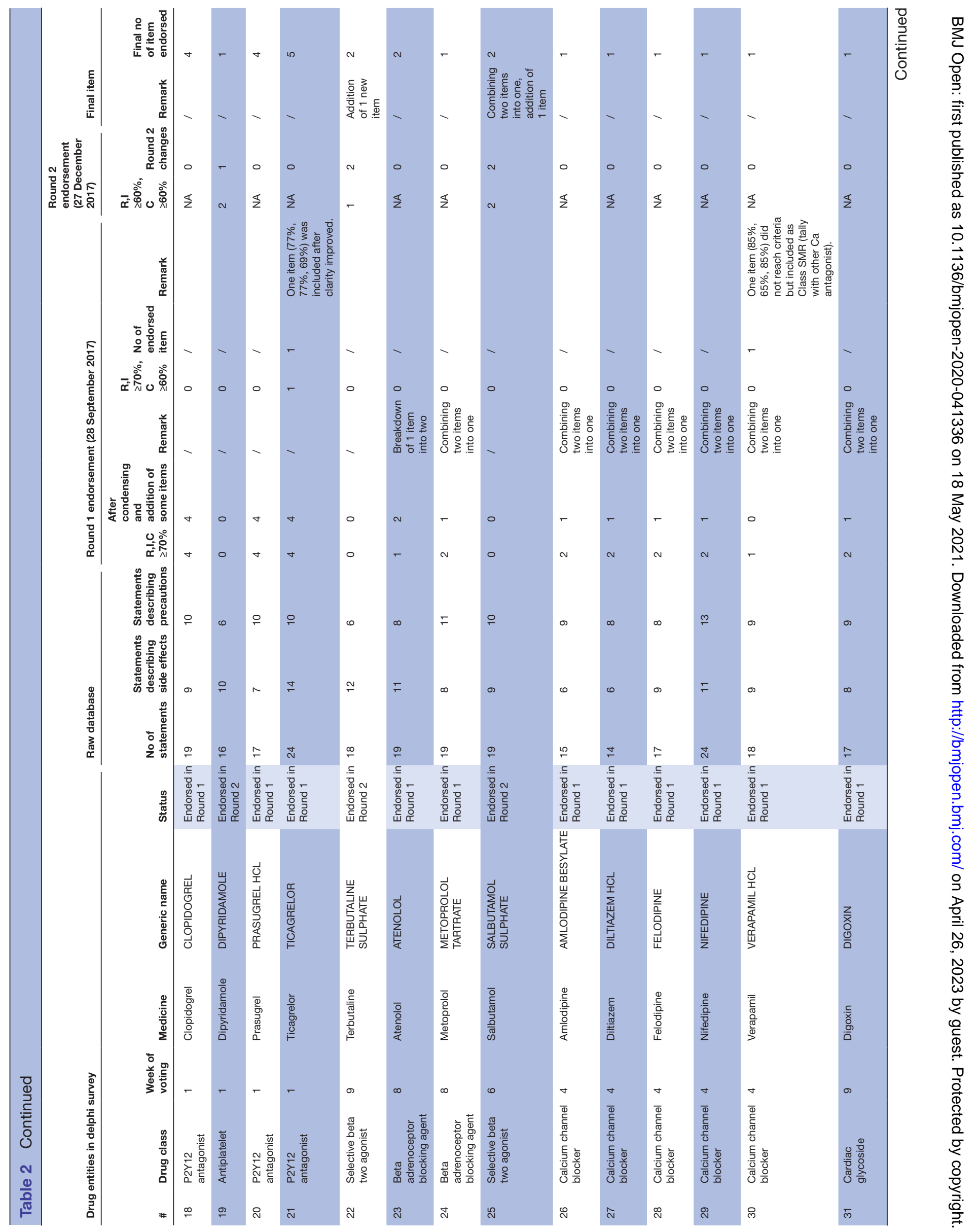




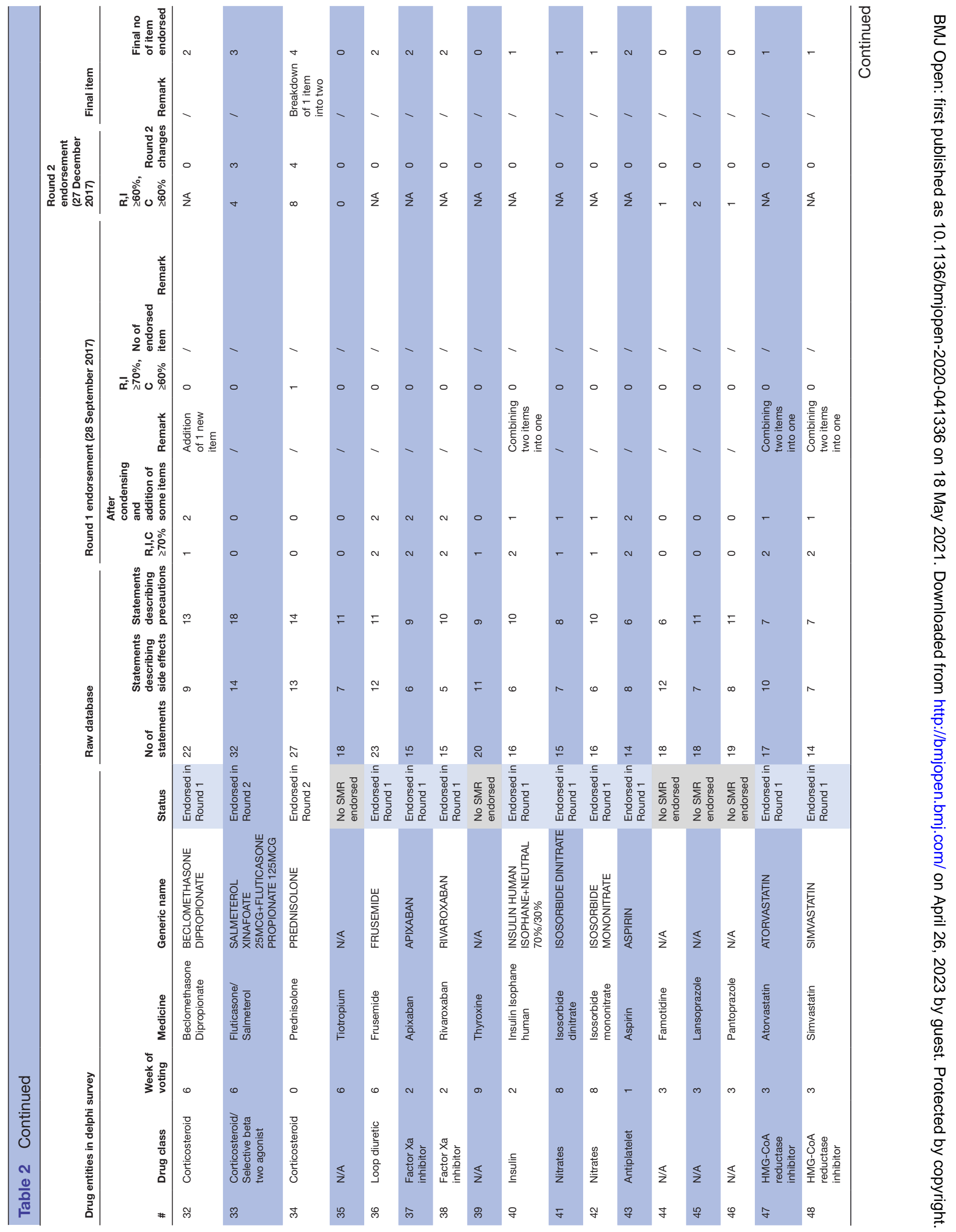




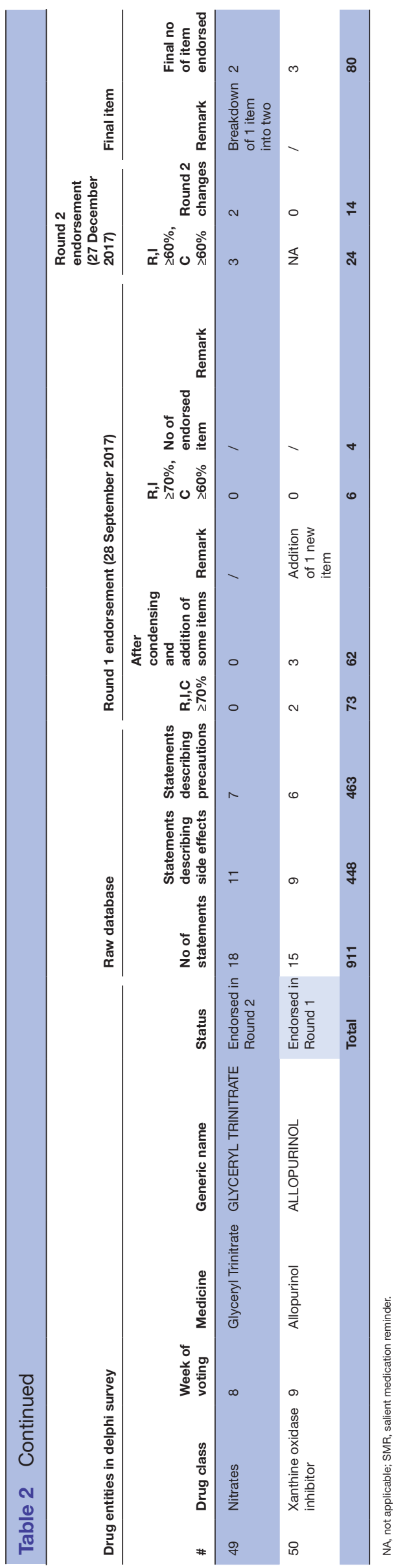

\section{Delphi survey and expert consensus discussion}

The steering group provided the expert panel with information on the study background, a list of 911 statements corresponding to 50 medication entities, statement voting criteria, and the details of the Delphi survey. Subsequently, the experts were invited to rate each statement on a scale from 1 (strongly disagree) to 5 (strongly agree) points, based on relevance, importance, and clarity, using an online platform. The statements were disseminated in 9weekly batches between April and July 2017.

A statement was accepted into the SMR framework if $\geq 70 \%$ of participants provided ratings of 4 (agree) or 5 (strongly agree) points on all three criteria. If $\geq 70 \%$ of participants provided ratings of $\geq 4$ points on statement relevance and importance, but $<70 \%$ of participants provided such ratings on clarity, the steering group made modifications based on the experts' suggestions and put a revised statement to a vote in the next round of the Delphi survey.

Moreover, statements with $60 \%-69 \%$ consensus and scores of 4-5 points on all three criteria were put to a faceto-face discussion before the next round of the Delphi survey. Revisions made to the statements by the steering group involved consolidation of pertinent statements, splitting lengthy statements and adding new statements, as required. The steering group discarded statements that failed to achieve agreement after two rounds of discussion. All consensus statements were endorsed by the steering group and included in the final list of SMR.

\section{Blinding}

The Delphi surveys were conducted using a doubleblind design. The panel participants were blinded to the responses obtained from the other participants to minimise response bias. Survey findings were aggregated, and only score distribution per statement was disclosed to the expert panel and steering group for discussion. Data analysis was conducted by two investigators who were blinded to the identity of each respondent.

\section{Statistical analysis}

For the Delphi process, the choice from the survey was recorded using descriptive statistics and qualitative feedback on criteria-related revisions was also recorded. A double-entry data input method was used to ensure accuracy. Statistical analyses were performed using Stata V.13.0, StataCorp. Descriptive statistics of the sampled demographics were presented as counts and percentages or mean values, as appropriate.

The entire survey was conducted anonymously, and the data were only accessible to assigned research team members to ensure confidentiality.

\section{RESULTS}

A panel comprising 13 experts participated in the Delphi process, including 9 consultants/deputy consultants and 4 associate consultants. Demographic characteristics of 
the participants are presented in table 1 . We achieved $100 \%$ response rates for both the Delphi survey and expert panel consensus discussion. Figure 2 provides details of the Delphi process results.

\section{Delphi survey}

Seventy-three $(8.0 \%$ of 911$)$ statements met the selection criteria. Additional $6(0.7 \%)$ statements were deemed relevant and important but not clear; finally, $832(81.6 \%)$ statements were deemed as neither relevant nor important. After voting, 73 statements remained. Thyroxine-related statements were excluded after discussion among the expert panel due to the lack of sufficient information on side effects; only 1 in 20 applicable statements achieved a consensus.

In addition, based on panel feedback, 26 statements were consolidated into 13 statements, 1 statement was split into 2 and another 2 statements were revised for clarity. Six statements deemed relevant and important but unclear were revised and included in the SMR. Among 832 statements with $<70 \%$ of votes on 'relevance' or 'importance', all expert panel agreed with the statement for 'verapamil' which was rated with $85 \%$ for 'relevance', $69 \%$ for 'importance' and $85 \%$ for 'clarity' in the Delphi survey should be also included in the SMR due to the high agreement regarding relevance after post Delphi survey discussion. A total of 66 statements on 38 medication entities achieved survey and discussion consensus for inclusion in the SMR. In addition, 24 statements on 6 medication entities that achieved $60 \%-69 \%$ consensus for both 'relevance' and 'importance' were revised and presented for further discussion.

A total of 196 comments on 911 statements were collected from the Delphi survey. Among them, 86 (44\%) comments were related to relevance (eg, 'side effect not relevant to older adults') and suggestions for other common side effects to be included; 21 (11\%) comments were about importance (eg, 'low risk of this side effect') and $78(40 \%)$ comments were about clarity and word choice (eg, 'not specific enough for the medication').

\section{Expert discussion}

Based on relevance, 11 of 24 statements were selected for further modification, and 13 statements were excluded. After reviewing the comments, 2 of 11 statements were consolidated. Statements related to prednisolone and glyceryl trinitrate were split from two into four statements. 'If symptoms persist, please consult a doctor' was used as a general reminder statement. A new statement, 'This medication relieves asthmatic symptoms; please consult a doctor if the symptoms persist', was suggested for salbutamol. Another new statement, 'Common side effects include palpitations, fine tremors and anxiety', was added for terbutaline. After discussion, all expert panel members agreed to include another 14 statements pertaining to 6 medication entities. Table 2 presents the drug entities considered in the Delphi process. Examples of the endorsed statements are presented in online supplemental table S1, including suggestions for modifications.

\section{Salient medication reminders}

After the Delphi process, a list of 80 statements on 44 medication entities covering 24 drug classes was compiled as the SMR framework for older adults discharged from hospital care (1) corticosteroids, (2) antiplatelet agents, (3) vitamin $\mathrm{K}$ antagonist, (4) insulins; (5) HMG-CoA reductase inhibitor, (6) P2Y12 antagonists, (7) biguanides, (8) calcium channel blockers, (9) penicillin, (10) selective beta-2 agonists, (11) antimuscarinic agents, (12) loop diuretics, (13) ACE inhibitors, (14) angiotensin-II receptor antagonist, (15) nitrates, (16) beta-adrenoceptorblocking agents, (17) alpha-adrenoceptor-blocking agents, (18) thrombin inhibitors (direct), (19) factor Xa inhibitor, (20) biguanides, (21) sulphonylureas, (22) xanthine oxidase inhibitor, (23) cardiac glycoside and (24) antihistamines (table 2).

The HA adopted a stepped-wedge design to launch an autogenerated patient discharge information summary, which was provided at discharge to patients aged $\geq 65$ years by the department of medicine, and included an SMR with the most relevant and important drug-related precautions, and a schedule of follow-up appointments at the HA. Staff were instructed to discuss the provided information with patients or caregivers to help increase their likelihood of self-care and reduce the risk of drugrelated adverse events associated with post-discharge errors. Patients or caregivers that required additional information could access it through hospital mobile apps.

\section{DISCUSSION}

In the present study, 13 experts from the fields of geriatric medicine, general medicine and pharmacy participated in the Delphi process, reaching a consensus on 80 statements regarding 44 medication entities to be included in the SMR for older adults discharged from acute care. The statements covered medications commonly dispensed to older adults at discharge from hospital in the local context, and included information on side effects and drug-related incidents that may be experienced after discharge. Patients and caregivers should be aware of the warning signs associated with adverse events while being treated at home or in an assisted living facility. The presented process may offer a framework for further development of information martials aimed at other populations or reporting on other medication entities.

Previous studies have shown that approximately half of all patients misunderstand at least one in five prescription labels. ${ }^{21}$ The type and quality of medication information differs between manufacturers and drug entities, presenting a need for standardisation of the type of information provided on commonly dispensed drugs. Using short, simple, and jargon-free statements may help patients understand important information on their regimen. ${ }^{22} 23$ The Delphi process may help identify, 
organise and revise statements to achieve relevance and clarity for the target audience.

'Improved discharge planning' has been a policy and research recommendation, aimed at streamlining the transition of care from hospital to community settings. Empowering patients to take charge of and actively participate in their care may help prevent medication-related harm. ${ }^{24}$ The present precautions related to 44 medication classes for older adults may be incorporated into the discharge process and patient education on drug availability and safety. The SMR may help initiate conversations and disseminate information, as well as encourage patients to voice their concerns and ask questions. ${ }^{25}$

The strengths of this study were the rigorous use of the Delphi process and the representativeness of stakeholders in both the steering group and expert panel. The participating experts differed in their work experience and field of expertise, minimising the risk of bias, which would have been high if only geriatric medicine experts were involved. This approach increased the internal validity of the present study.

A limitation of this study was the adoption of consensus discussion to reach the final agreement, instead of voting in a Delphi survey. This approach may have given undue weight to the views of some experts; however, the steering group provided opportunities for the other experts to express their concerns, as required. Another limitation of the present study is the collection of feedback, especially regarding medication reminders from other key stakeholders, such as different groups of patients and nurses was not included, because they are users and distributors, respectively. Their views should be considered alongside those from doctors and pharmacists to improve the $\mathrm{SMR}^{26}$

\section{CONCLUSIONS}

Regimen prescriptions should be accompanied by information that may improve patient knowledge, awareness and experience, concurrently, increasing the rates of medication safety and efficacy. Although patients have access to their complete discharge data, the amount and complexity of this information, in particular, in cases of polypharmacy, can be overwhelming to patients and their caregivers. SMRs may help communicate the key precautions and improve the likelihood of desirable treatment outcomes.

\section{Author affiliations}

${ }^{1}$ Centre for Health Systems and Policy Research, JC School of Public Health and Primary Care, Faculty of Medicine, The Chinese University of Hong Kong, Hong Kong, Hong Kong

${ }^{2}$ Quality and Safety Division, New Territories West Cluster, Hong Kong Hospital Authority, New Territories, Hong Kong

${ }^{3}$ Department of Medicine and Geriatrics, Tuen Mun Hospital, Hong Kong Hospital Authority, Hong Kong, Hong Kong

${ }^{4}$ Department of Medicine, Pamela Youde Nethersole Eastern Hospital, Hong Kong Hospital Authority, Hong Kong, Hong Kong

${ }^{5}$ Department of Medicine and Geriatrics, Kwong Wah Hospital, Hong Kong Hospital Authority, Hong Kong, Hong Kong
${ }^{6}$ Department of Medicine, Tseung Kwan 0 Hospital, Hong Kong Hospital Authority, Hong Kong, Hong Kong

${ }^{7}$ Department of Medicine and Geriatrics, Tai Po Hospital, Hong Kong Hospital Authority, Hong Kong, Hong Kong

${ }^{8}$ Department of Medicine \& Geriatrics, Caritas Medical Center, Hong Kong Hospital Authority, Hong Kong, Hong Kong

${ }^{9}$ Department of Medicine, North District Hospital, Hong Kong Hospital Authority, Hong Kong, Hong Kong

${ }^{10}$ Department of Medicine, Tung Wah Group of Hospitals Fung Yiu King Hospital, Hong Kong Hospital Authority, Hong Kong, Hong Kong

${ }^{11}$ Department of Medicine and Geriatrics, United Christian Hospital, Hong Kong Hospital Authority, Hong Kong, Hong Kong

Acknowledgements We are grateful to the members of the steering committee for professional advice regarding implementation of PDIS. We acknowledge the assistance of Ms. Tang PF. We would also like to thank HA Head Office for their kind assistance in the logistics for conducting the study.

Contributors ELYW, KST, SFL and EKY conceived the study design. ELYW and KST were the project in-charge to lead the study and transfer the study findings to the application in healthcare. FCKM, PWY, JYKC, WCL, SKM, TYC, SWCT, JSWL, MMLW, CSL, KHC, JKHL and SYF were members of the expert panel that provided professional advice in the expert discussion and valuable insights on the development of medication safety reminders. ELYW, AWLC, RKCS and JCHL extracted the data and conducted the analysis with input from all authors. AWLC, RKCS and JCHL were responsible for the finding presentation in visual aids. ELYW drafted the manuscript and all authors edited the manuscript. All authors read and approved the final manuscript.

Funding The authors have not declared a specific grant for this research from any funding agency in the public, commercial or not-for-profit sectors.

Competing interests None declared.

Patient consent for publication Not required.

Ethics approval The present study was conducted in collaboration with the Hong Kong HA. The study protocol was approved by the Survey and Behavioural Research Ethics Committee of the Chinese University of Hong Kong (003-2017).

Provenance and peer review Not commissioned; externally peer reviewed.

Data availability statement Data sharing not applicable as no datasets generated and/or analysed for this study.

Supplemental material This content has been supplied by the author(s). It has not been vetted by BMJ Publishing Group Limited (BMJ) and may not have been peer-reviewed. Any opinions or recommendations discussed are solely those of the author(s) and are not endorsed by BMJ. BMJ disclaims all liability and responsibility arising from any reliance placed on the content. Where the content includes any translated material, BMJ does not warrant the accuracy and reliability of the translations (including but not limited to local regulations, clinical guidelines, terminology, drug names and drug dosages), and is not responsible for any error and/or omissions arising from translation and adaptation or otherwise.

Open access This is an open access article distributed in accordance with the Creative Commons Attribution Non Commercial (CC BY-NC 4.0) license, which permits others to distribute, remix, adapt, build upon this work non-commercially, and license their derivative works on different terms, provided the original work is properly cited, appropriate credit is given, any changes made indicated, and the use is non-commercial. See: http://creativecommons.org/licenses/by-nc/4.0/.

ORCID iD

Eliza Lai-Yi Wong http://orcid.org/0000-0001-9983-6219

\section{REFERENCES}

1 Angamo MT, Chalmers L, Curtain CM, et al. Adverse-DrugReaction-Related hospitalisations in developed and developing countries: a review of prevalence and contributing factors. Drug Saf 2016;39:847-57.

2 Parekh N, Ali K, Page A, et al. Incidence of medication-related harm in older adults after hospital discharge: a systematic review. J Am Geriatr Soc 2018;66:1812-22.

3 Barber N, Parsons J, Clifford S, et al. Patients' problems with new medication for chronic conditions. Qual Saf Health Care $2004 ; 13: 172-5$ 
4 Taché SV, Sönnichsen A, Ashcroft DM. Prevalence of adverse drug events in ambulatory care: a systematic review. Ann Pharmacother 2011;45:977-89.

5 Ellins J, Glasby J, Tanner D. Understanding and improving transitions of older people: a user and carer centred approach. Final report National Institute for Health Research Service Delivery and Organisation programme; 2012.

6 Williams $\mathrm{H}$, Edwards $\mathrm{A}$, Hibbert $\mathrm{P}$, et al. Harms from discharge to primary care: mixed methods analysis of incident reports. $\mathrm{Br} J \mathrm{Gen}$ Pract 2015;65:e829-37.

7 Riordan Ciara O', Delaney T, Grimes T. Exploring discharge prescribing errors and their propagation post-discharge: an observational study. Int J Clin Pharm 2016;38:1172-81.

8 Forster AJ, Murff HJ, Peterson JF, et al. Adverse drug events occurring following hospital discharge. J Gen Intern Med 2005;20:16:317-23.

9 Coleman EA, Berenson RA. Lost in transition: challenges and opportunities for improving the quality of transitional care. Ann Intern Med 2004;141:533-6.

10 Ensing HT, Koster ES, van Berkel PI, et al. Problems with continuity of care identified by community pharmacists post-discharge. J Clin Pharm Ther 2017;42:170-7.

11 Census and Statistics Department. Thematic household survey report No. 40 socio-demographic profile, health status and self-care capability of older persons. Hong Kong Social Surveys Section Census and Statistics Department; 2009.

12 Lam PW, Lum CM, Leung MF. Drug non-adherence and associated risk factors among Chinese geriatric patients in Hong Kong. Hong Kong Med J 2007;13:284-92.

13 Lee VWY, Pang KKW, Hui KC, et al. Medication adherence: is it a hidden drug-related problem in hidden elderly? Geriatr Gerontol Int 2013;13:978-85.

14 Hong Kong Hospital Authority. Patient experience and satisfaction survey on inpatient service (20169). Hong Kong; 2015.
15 Yam CHK, Wong ELY, Chan FWK, et al. Avoidable readmission in Hong Kong--system, clinician, patient or social factor? BMC Health Serv Res 2010;10:311.

16 Mistiaen P, Francke AL, Poot E. Interventions aimed at reducing problems in adult patients discharged from hospital to home: a systematic meta-review. BMC Health Serv Res 2007;7:47.

17 Helmer O. Analysis of the future: the Delphi method in DTIC document 1967.

18 Trevelyan EG, Robinson PN. Delphi methodology in health research: how to do it? Eur J Integr Med 2015;7:423-8.

19 Habibi A, Sarafrazi A, Izadyar S. Delphi technique theoretical framework in qualitative. Int J Eng Sci 2014;3:8-13.

20 Clauson KA, Marsh WA, Polen $\mathrm{HH}$, et al. Clinical decision support tools: analysis of online drug information databases. BMC Med Inform Decis Mak 2007;7:7.

21 Davis TC, Wolf MS, Bass PF, et al. Literacy and misunderstanding prescription drug labels. Ann Intern Med 2006;145:887-94. vol.

22 Bailey SC, Navaratnam P, Black H, et al. Advancing best practices for prescription drug labeling. Ann Pharmacother 2015;49:1222-36.

23 Mullen RJ, Duhig J, Russell A, et al. Best-practices for the design and development of prescription medication information: a systematic review. Patient Educ Couns 2018;101:1351-67.

24 Yam CHK, Wong ELY, Cheung AWL, et al. Framework and components for effective discharge planning system: a Delph methodology. BMC Health Serv Res 2012;12:396.

25 Linn AJ, van Weert JC, Schouten BC, et al. Words that make pills easier to swallow: a communication typology to address practica and perceptual barriers to medication intake behavior. Patient Prefer Adherence 2012;6:871-85.

26 Hohmann E, Brand JC, Rossi MJ, et al. Expert opinion is necessary: Delphi panel methodology facilitates a scientific approach to consensus. Arthroscopy 2018;34:349-51. 